\title{
miR-129-5p inhibits oxidized low-density lipoprotein-induced A7r5 cells proliferation and migration through targeting HMGB1 involving a PI3K/Akt signal pathway
}

Hongfei Jiang

Nanchang University Second Affiliated Hospital

Ren Gong

Nanchang University Second Affiliated Hospital

Yanqing Wu ( $\sim$ wuyanqing01@sina.com )

Nanchang University Second Affiliated Hospital https://orcid.org/0000-0002-3042-271X

\section{Research}

Keywords: miR-129-5p, A7r5 cells, HMGB1, proliferation

Posted Date: October 26th, 2020

DOI: https://doi.org/10.21203/rs.3.rs-34905/v1

License: (c) (i) This work is licensed under a Creative Commons Attribution 4.0 International License.

Read Full License 


\section{Abstract \\ Background}

Currently, gene therapy for cardiovascular diseases has been widely concerned, but its mechanism is still unclear.

\section{Objective}

Recently miRNAs have been recognized as a key regulator in vascular smooth muscle cells (VSMCs) which involved in the formation of atherosclerosis. The aim of the study was to explore the role of miR129-5p in regulation of HMGB1 involving a PI3K/Akt signal pathway as well as the proliferation and migration in $A 7 r 5$ cells induced by ox-LDL.

\section{Methods}

Cell viability, proliferation and migration were conducted by CCK-8, colony formation, wound healing assay and transwell assay. The expressions of miR-129-5p and HMGB1 were detected by real-time quantitative-qPCR (RT-qPCR) and western blot. Luciferase assay was used to confirm that miR-129-5p directly targeted HMGB1.

\section{Results}

The expression of miR-129-5p in A7r5 cells induced by ox-LDL was significantly decreased in comparison with the control cells. Cell viability, proliferation and migration of A7r5 cells induced by ox-LDL were increased. MiR-129-5p could down-regulate the expression of HMGB1 in A7r5 cells. More studies showed that miR-129-5p could inhibit cell viability, proliferation on and migration of A7r5 cells induced by ox-LDL and target HMGB1 to regulate PI3K/Akt signal pathway.

\section{Conclusion}

miR-129-5p could inhibit PI3K/Akt signal pathway by target HMGB1 and further restrain the cell viability, proliferation and migration of $A 7 r 5$ cells induced by ox-LDL.

\section{Background}

Cardiovascular disease (CVD) is the leading cause of death throughout the world. One of the most major causes of CVD is atherosclerosis (AS) [1], whose pathological mechanism has attracted extensive attention in recent decades. AS is now recognized as a chronic arterial inflammatory disease that is 
induced by oxidized low-density lipoprotein (ox-LDL) accumulation and inflammation in the arterial intima under hypercholesterolemic conditions. Modern pharmacological research have shown that the main risk factors of atherosclerosis include dyslipidemia, hypertension, drinking, smoking, diabetes, obesity and lack of exercise. Whereas among the factors, dyslipidemia is commonly considered as the most critical one. Ox-LDL is a critical marker of atherosclerosis [2], which could cause lipid metabolism disorder, promote the formation of foam cells derived from vascular smooth muscle cells (VSMCs), and regulate the proliferation, apoptosis, migration and differentiation of VSMCs, which play vital roles in the development of atherosclerosis disease [3].

Studies in the past decades have revealed the key signals and molecular pathways that underlie the formation and development of atherosclerotic plaques. Recently, the researches on the role of miRNA in cardiovascular diseases have attracted extensive attention. MicroRNAs (miRNAs) are a family of highly conserved, endogenous non-coding small RNA molecules that regulate gene expression through combining with the $3^{\prime}$-untranslaed region (3'-UTR) of the target mRNAs $[4,5,6]$. MiRNAs are involved in diverse cellular functions, including differentiation, growth, proliferation, migration, senescence and apoptosis in various cells [7]. Moreover, previous studies have shown that miRNAs play essential roles in regulating atherosclerosis [8]. MicroRNAs, as important regulators of pathophysiological processes have provided novel molecular insights into their impact on these pathways in atherosclerosis and identified new therapeutic targets. For instance, miR-181b as an inhibitor of endothelial inflammatory responses by targeting NF-KB signaling in atherosclerosis disease [9]. In vitro studies have implicated miR-221/miR-222 in regulating PDGF-mediated VSMC proliferation [10]. MiR-145 were proved to play a regulative role in aberrant VSMC proliferation, which is a key pathological process of atherosclerosis [11]. Liu YR et al proved that miR-21 is an important target for protective reagent against ox-LDL induced rat vascular endothelial cells (VECs) injury, which may play critical roles in development of atherosclerosis [12]. Among all miRNAs, miR-129-5p has been shown to suppress carcinogenesis of various cancers $[13,14]$. Nevertheless, a role of miR-129-5p in the atherosclerosis has not been further explored. High-mobility group box 1 protein (HMGB1), a highly conserved and widely expressed DNA-binding protein, is a key mediator of cell migration and proliferation [15]. Interestingly, recent evidence demonstrated that HMGB1 was overexpressed in cancer cells and promoted cell invasion and migration in vitro [16].

In the present study, we utilized ox-LDL induced A7r5 cells to detect miR-129-5p to inhibit cell migration and proliferation by targeting the HMGB1 involving signaling pathway $\mathrm{PI} 3 \mathrm{~K} / \mathrm{Akt}$.

\section{Results}

\section{Ox-LDL increases proliferation and migration of A7r5 cells}

Firstly, we aimed to study the effect of the proliferation activity of A7r5 cells induced by ox-LDL. Cell counting kit-8 (CCK-8) was used to detect the proliferation of A7r5 cells induced by $0,10,20,40 \mu \mathrm{g} / \mathrm{mL}$ ox-LDL. After ox-LDL treatment, CCK-8 results showed that the index of A7r5 cells significantly increased with the increase of ox-LDL concentration, indicating that ox-LDL increased proliferation in a dose- 
dependent manner (Fig. 1A). As shown in Fig. 1, A7r5 cells induced by $40 \mu \mathrm{g} / \mathrm{mL}$ ox-LDL showed the best proliferation activity. Clonoy formation was used to detect the proliferation of $A 7 r 5$ cells induced by 0,10 , $20,40 \mu \mathrm{g} / \mathrm{mL}$ ox-LDL. As exhibited in Fig. 1B, we found that the index of A7r5 cells significantly increased with the increase of ox-LDL concentration, indicating that $0 x-L D L$ increased proliferation in a dosedependent manner and $A 7 \mathrm{r} 5$ cells induced by $40 \mu \mathrm{g} / \mathrm{mL}$ ox-LDL showed the best proliferation activity. Clone formation reflects the best proliferation ability of A7r5 cells induced by $40 \mu \mathrm{g} / \mathrm{mL}$ ox-LDL. At the same time, wound healing and transwell results reflect the best migration ability of A7r5 cells induced by $40 \mu \mathrm{g} / \mathrm{mL}$ ox-LDL (Fig. 1CD)

\section{MiR-129-5p decreases and HMGB1 increases during A7r5 cells induced by ox-LDL}

The qRT-PCR results showed that the level of miR-129-5p decreases with the increase of ox-LDL in A7r5 cells. Compare to the control group, the level of miR-129-5p was the lowest in A7r5 cells induced by 40 $\mu \mathrm{g} / \mathrm{mL}$ ox-LDL. (Fig. 2A, ${ }^{* * *} P<0.001$ ). Furthermore, the expression mRNA level and protein level of HMGB1 in A7r5 cells induced by different doses of ox-LDL was evaluated via western blot and qRT-PCR. As exhibition in Fig. 2B, CD, compare to the control group, both the protein level and mRNA level of HMGB1 were the highest in $A 7 r 5$ cells induced by $40 \mu \mathrm{g} / \mathrm{mL}$ ox-LDL $\left({ }^{* *} P<0.001\right)$. It means the level of HMGB1 was increased in A7r5 cells induced by ox-LDL and miR-129-5p was decreased.

\section{MiR-129-5p regulates HMGB1 expression by binding its 3' UTR}

Targetscan and miR Base suggest that 3'UTR of HMGB1 contained the miR-129-5p seed sites (Fig. 3A). In order to detect whether miR-129-5p directly target HMGB1, double-luciferase analysis was performed after A7r5 cells were co-transfected with HMGB1-WT or HMGB1-mut reporter plasmids and miR-129-5p or miR-NC respectively. The results showed that miR-129-5p down-regulated the luciferase activity of HMGB1-WT plasmid ( ${ }^{* \star *} P<0.001$, Fig. 3B), but had no effects on that of HMGB1-mut plasid $(P>0.05)$. The results showed that miR129-5p directly targeted HMGB1. In addition, western blot and qRT-PCR results showed the differences of the expression level of HMGB1 in A7r5 cells with miR-129-5p over-expressed. As shown in Fig. 3C.D.E, miR-129-5p could down-regulated the protein level and mRNA level of HMGB1 in A7r5 cells.

\section{MiR-129-5p inhibits proliferation and migration of A7r5 cells induced by ox-LDL}

QRT-PCR analysis was conducted to detect the mRNA level of miR-129-5p in A7r5 cells induced by oxLDL. Compare to the control group, the level of miR-129-5p in A7r5 cells induced by $40 \mu \mathrm{g} / \mathrm{mL}$ ox-LDL was lower significantly ( ${ }^{* *} P<0.001$, Fig. 4A). In contrast with that in the ox-LDL group, the level of the group with miR-129-5p over-expressed increased significantly ( ${ }^{\star * *} P<0.001$, Fig. 4A). CCK-8 assay was conducted to analyze the viability of A7r5 cells induced by $40 \mu \mathrm{g} / \mathrm{mL}$ ox-LDL (Fig. 4B). Compare to the control group, the viability of $A 7 \mathrm{r} 5$ cells induced by $40 \mu \mathrm{g} / \mathrm{mL}$ ox-LDL increased more. Otherwise $\bigotimes$ the viability of A7r5 cells with miR-129-5p over-expressed induced by $40 \mu \mathrm{g} / \mathrm{mL}$ ox-LDL was lower $\left({ }^{* *} P<0.001\right)$. Similarly, colony formation and transwell were performed to identify the proliferation and 
migration of $A 7 r 5$ cells induced by ox-LDL. The results showed that the proliferation and migration of A7r5 cells induced by $40 \mu \mathrm{g} / \mathrm{mL}$ ox-LDL were increased. However, the proliferation and migration of the cells with miR-129-5p over-expressed induced by $40 \mu \mathrm{g} / \mathrm{mL}$ ox-LDL were decreased significantly $\left({ }^{\star * \star} P<0.001\right.$, Fig. 4C, D).

The overexpression of HMGB1 affected the results of miR-129-5p inhibiting proliferation and migration of A7r5 cells induced by ox-LDL

QRT-PCR was conducted to detect the mRNA level of HMGB1 in A7r5 cells induced by ox-LDL or miR-129$5 p$ over-expressed. Compare to the control group, the level of HMGB1 in A7r5 cells induced by $40 \mu \mathrm{g} / \mathrm{mL}$ ox-LDL was lower significantly $\left({ }^{\star * *} P<0.001\right.$, Fig. 5 A). In contrast with that in the miR-129-5p overexpressed group, the level of the group with HMGB1 over-expressed increased significantly $\left({ }^{\star \star *} P<0.001\right.$, Fig. 5 A). CCK-8 assay was conducted to analyze the viability of A7r5 cells induced by $40 \mu \mathrm{g} / \mathrm{mL}$ ox-LDL (Fig. 5 B). Compare to the control group, the viability of A7r5 cells with miR-129-5p over-expressed increased more. Otherwise the viability of A7r5 cells with HMGB1 over-expressed induced by $40 \mu \mathrm{g} / \mathrm{mL}$ ox-LDL was lower $\left({ }^{\star \star *} P<0.001\right)$. Similarly, colony formation and transwell were performed to identify the proliferation and migration of A7r5 cells induced by ox-LDL. The results showed that the proliferation and migration of $A 7 \mathrm{r} 5$ cells induced by $40 \mu \mathrm{g} / \mathrm{mL}$ ox-LDL were increased. However, the proliferation and migration of the cells with HMGB1 over-expressed induced by $40 \mu \mathrm{g} / \mathrm{mL}$ ox-LDL were increased significantly $\left({ }^{\star \star *} P<0.001\right.$, Fig. 5CD).

\section{The effect of miR-129-5p on the expression and phosphorylation of FAK and Akt}

The PI3K/Akt-related proteins in A7r5 cells were investigated with miR-129-5p over expressed or induced by $40 \mu \mathrm{g} / \mathrm{mL}$ ox-LDL. As shown in Fig. 6, the protein level of PI3k and phosphorylation level of Akt and FAK in A7r 5 cells induced by $40 \mu \mathrm{g} / \mathrm{mL}$ ox-LDL were higher than that of the control group. Whereas, the level of PI3k and phosphorylation level of Akt and FAK in A7r5 cells with miR-129-5p over-expressed were lower that that of the group with $40 \mu \mathrm{g} / \mathrm{mL}$ ox-LDL $\left({ }^{\star \star *} P<0.001\right.$, Fig. 6).

\section{Discussion}

Atherosclerosis is the cause of cardiovascular and cerebrovascular diseases, which leads to the highest mortality and morbidity in the world. The main risk factors for atherosclerosis include hyperglycemia, hyperlipidemia, smoking, high calories, obesity and diabetes mellitus. Epidemiological data showed that the mortality rate of cardiovascular diseases is much higher than that of other diseases worldwide. Vascular smooth muscle cell proliferation is one of the major contributors to atherosclerosis, thus its risk factors are being extensively studied. One of them is $0 x-L D L$, a result of $0 x-L D L$ oxidation $[18,19,20]$. In addition, efforts to improve and prevent atherosclerosis are continuing. Hyperlipidemia, especially ox-LDL has been shown to be an important risk factor in the development of atherosclerosis [21]. In the last decades, the pharmacological inhibition of miRNAs to treat human diseases continuing to show promise, and, there has been a trend in the development of RNA-based therapeutics in clinical application, 
particularly cardiovascular disease. As one of the most important cells in the arterial mesangium, vascular smooth muscle cells (VSMCs) play a key role in the formation of arteriosclerosis [22, 23]. As an important role, the excessive proliferation of smooth muscle cells is a key factor leading to atherosclerosis. Several studies have utilized VSMCs as a model to elucidate the pathological mechanism of atherosclerosis. MiRNAs have become an integral part in determining the mechanisms associated with atherosclerosis that is aberrantly expressed and also act as potential biomarkers [24, 25]. Hence, we speculate that miRNA may could regulate proliferation, migration and cell viability of A7r 5 cells induced by ox-LDL.

To understand the functional mechanism of microRNAs, identifying targets involved in their regulation is important. Luo et al. reported that miR-129-5p attenuates irradiation-induced autophagy and decreases radio-resistance of breast cancer cells by targeting HMGB1 $[8,26,27]$. In the study, we sought to investigate the role of miR-129-5p on atherosclerosis and the possible molecular mechanism. We have performed the experiments to detect the effects of miR-129-5p on the proliferation and migration of A7r5 cells induced by ox-LDL. In addition, HMGB1 is well known as one of the targets of PI3k/Akt signaling pathway. And our results showed that miR-129-5p regulates PI3K/Akt signaling pathway targeting HMGB1. From our results, as an important regulator of PI3K/Akt signal pathway in VSMCs, HMGB1 was further identified as a direct functional target of miR-129-5p in A7r5 cells. For this identification, we firstly showed that the 3'UTR of HMGB1 contained a binding site that matched the miR-129-5p seed sequence. Besides, overexpression of miR-129-5p decreased the luciferase activity upstream of the wild type 3' UTR of HMGB1, whereas a site mutation to miR-129-5p abolished miR-129-5p regulation. And, inhibition of miR-129-5p led to the increasing of luciferase activity of wild type HMGB1 3'UTR in A7r5 cells. Finally, transfection of the miR-129-5p inhibitor suppressed HMGB1 expression at the translational level. Therefore, we found that miR-129-5p regulated HMGB1 expression by directly binding to its 3 'UTR (Fig. 3).

An accumulating evidences indicate the possible roles of HMGB1 in VSMCs. It is the reason that the proteins are specific regulator in PI3K/Akt signaling pathway and are vital pathways central to the development or progression of atherosclerosis, such as cell growth, migration, apoptosis of A7r5 cells induced by ox-LDL. The effective inhibition of VSMC proliferation at the early stage of AS formation is an important method for the prevention and treatment of vascular hyperplastic lesions and restenosis after angioplasty.

\section{Conclusion}

Considering that miR-129-5p mediates HMGB1 to regulate the PI3K/Akt signaling pathway and further inhibits the proliferation and migration of $A 7 r 5$ cells induced by ox-LDL, it is suggested that our study would provide a new sight to treat atherosclerosis by regulating proliferation and migration of VSMCs.

\section{Materials And Methods}




\section{Materials}

Fetal bovine serum (FBS), newborn calf serum, and endothelial cell growth supplement were supplied by Gibco (CA, USA). Ox-LDL was purchased from Peking Union-Biology Co. Ltd. (Beijing, China). Cell counting kit-8 (CCK-8) was purchased from Dojindo China (Cat. Number CK04, Shanghai, China). FAK (Cell Signaling Technology,CST\#3285), PI3K (Cell Signaling Technology, CST\#4255), Akt (Cell Signaling Technology, CST\#9272), p-FAK (Cell Signaling Technology, CST\#3281), p-Akt (Cell Signaling Technology, CST\#9275).

\section{Cell culture and treatment}

Cell culture

The A7r5 cells were purchased from American Type Culture Collection (ATCC, USA) and maintained in Dulbecco's modified Eagle's medium (DMEM; Gibco, USA) containing 10\% fetal bovine serum (FBS; Hyclone, Logan, UT, USA), $1 \mathrm{mmol} / \mathrm{L}$ sodium pyruvate, $4 \mathrm{mmol} / \mathrm{L} \mathrm{L}$-glutamine, $4.5 \mathrm{~g} / \mathrm{L}$ glucose, $1.5 \mathrm{~g} / \mathrm{L}$ sodium bicarbonate, $100 \mathrm{mg} / \mathrm{mL}$ streptomycin and $100 \mathrm{U} / \mathrm{ml}$ penicillin, at $37^{\circ} \mathrm{C}$ in a humidified atmosphere with $5 \% \mathrm{CO}_{2}$.

\section{Cell transfection}

The A7r5 cells were transfected with the miR-129-5p mimics or negative control (Shanghai Genomic Pharmaceutical Co., LTD., Shanghai, China) using lipofectamine 2000 reagent (Invitrogen, Carlsbad, CA) for 48 hours.

\section{Wound healing}

A7r5 cells were placed in a 6-well culture plates $\left(1 \times 10^{6}\right.$ cells/well) for $48 \mathrm{~h}$. The wound healing assay was conducted as described previous study [17]. The cell monolayers on the surface of the 6-well plate were scratched with the tip of 200 micropipette. Non-adherent cells were washed with PBS and the remaining cells were treated with ox-LDL $(0,10,20,40 \mu \mathrm{g} / \mathrm{mL})$. Cells were photographed with a $40 \times$ objective lens, and images of linear wounds were taken at 9 fields per well from 0 to $48 \mathrm{~h}$ after injury. Three independent repeated experiments were carried out.

\section{CCK-8 assays for detection of cell proliferation}

Cell proliferation was assessed with a Cell Counting Kit-8 (CCK-8) assay kit (Dojindo, Japan). A7r5 cells were separated cultured in 96-well plates overnight at a density of $10^{4}$ cells/well then transfected with miR-129-5p mimics or an inhibitor as described above. At $48 \mathrm{~h}$ after transfection, $10 \mu \mathrm{L}$ of CCK-8 solution was added to each well for $1 \mathrm{~h}$ and absorance reading at $450 \mathrm{~nm}$ were obtained in triplicate using a spectrophotometric plate reader. Three duplicate wells were measured for each data point to obtain data. Three separate experiments were conducted. 


\section{Transwell migration assay}

A7r5 cells were were harvested by trypsinization. Cells $\left(1 \times 10^{5}\right)$ were placed into transwell chambers (Corning Incorporated, USA) for migration assay, and $1 \times 10^{4}$ cells were placed into upper chambers coated with $150 \mathrm{mg}$ matrigel for invasion. The lower chambers were filled with DMEM containing $10 \%$ FBS. After incubation at $37^{\circ} \mathrm{C}$ for $48 \mathrm{~h}$, cells remaining on the upper surface of the membrane were removed. Cells on the lower surface of the membrane were fixed and stained with crystal violet. Then stained cells were observed and counted under a light microscope. Three independent experiments were performed.

\section{Colony formation assay}

A7r5 cells were treated with ox-LDL $(0,10,20,40 \mu \mathrm{g} / \mathrm{mL})$ and seeded in $60 \mathrm{~mm}$ plates at 1000 cells/well and cultured for another 14 days to form colonies. Cells were washed with $1 \times$ PBS, fixed in methanol and stained with $10 \%(w / v)$ Giemsa. Afterwards, colonies were viewed and counted under a microscope (Nikon Corp., Tokyo, Japan), and the pictures were taken with a digital camera (Canon, Tokyo, Japan). The percentage of colony formation for each group was calculated with the equation: percentage of colony formation $(\%)=$ the number of colony $/ 1000 \times 100$. Three independent experiments were performed.

\section{RNA isolation and qRT-PCR for miR-129-5p}

SYBR Green-based real-time quantification of miRNAs was used to determine miR-129-5p expression as previously described. Total RNA was extracted using the Trizol reagent (Invitrogen). Then, qRT-PCR was performed using SYBR Green mix with primers specific to miR-129-5p (RIBOBIO, Guangzhou, China). Relative quantification of the miRNA expression was calculated with the $2^{-\triangle \triangle C T}$ method. The corresponding PCR primers were as follows: 5'-ACA CTC CAG CTG GGT CCC TGA GAC CCT TAA-3' and CTCAAC TGG TGT GGA GT-3) for miR-129-5p; 5'-CTC GCT TCG GCA GCA CA-3' and 5'-AAC GCT TCA CGA AYY YGC GT-3' for U6. U6 was selected as the housekeeping gene to normalize the expression of miR125a-5p.

\section{Quantitative Real-Time Polymerase Chain Reaction (qRT-PCR)}

QRT-PCR analysis were performed by standard techniques. Briefly, total RNA extraction from A7r5 cells was conducted with the Trizol reagent kit according to the manufactures's instructions. Real-time quantitative PCR analysis was performed to detect the expression of miR-129-5p and HMGB1. The relative expression levels were calculated with the $2^{-\Delta \Delta C T}$ method and three independent experiments were performed.

\section{Luciferase activity assay}

WT or MUT of miR-129-5p was subcloned into pGL3 Basic vector (Promega, Madison, WI). Mimics of miR-129-5p (RiboBio, Guangzhou, China) were co-transfected with pLUC-WT-HMGB1 or pLUC-MUT- 
HMGB1 into A7r5 cells. Luciferas eactivity was tested using the Dual-Luciferase Reporter Assay System (Promega). Renilla luciferase was employed for normalization. Relative luminescence was tested by luminometry.

\section{Western blotting assay}

The cell lysates from ox-LDL-treated A7r5 cells were placed on ice in 1×RIPA lysis buffer (Sigma, US) containing protease and phosphatase inhibitor (Thermo-Scientific, Waltham, MA, USA). The concentration of the protein in cells lysates was detected using a BCA kit (Beijing solarbio science \& technology co., LTD., China). The proteins in cell lysates $(10 \mu \mathrm{g})$ were separated on $10 \%$ SDS-PAGE gels and electro-transferred to a nitrocellulose membrane. The membrane was incubated with $5 \%$ non-fat milk solution or BSA for $1 \mathrm{~h}$ at room temperature. Subsequently, the membranes were incubated with primary antibodies for HMGB1, FAK, Akt, p-FAK, p-Akt and GAPDH at $4^{\circ} \mathrm{C}$ overnight. The membrane was extensively washed with TBS-T and then incubated with anti-rabbit IgG (or anti-mouse IgG) antibody (Beijing zhongshan jinqiao biotechnology co., LTD) conjugated to HRP for $1 \mathrm{~h}$ at room temperature. Then the bands were visualized using an ECL detection kit. Then band intensities were determined densitometrically with image $\mathrm{J}$ software, and normalized to GAPDH intensity.

\section{Statistical Analysis}

Each experiment was carried out at least three times, and all values were represented as means \pm SD. Comparisons between two means were evaluated with the unpaired Student's t-test, and comparisons between three (or more) means were evaluated via ANOVA with Dunnett's posttests analysis. A value of $\mathrm{P}<0.05$ was considered statistically significant.

\section{Abbreviations}

No applicable.

\section{Declarations}

\section{Ethics approval and consent to participate:}

We have stated that Ethics approval and consent to participate: No applicable.

Consent for publication: If and when the manuscript was accepted for publication, all co-authors agree to publish the journal.

Competing interests: All authors declare that there is no any conflict of interest.

Funding: We are grateful to all participates for their contributions for the present study. This study was supported by the funded project of National Natural Science Foundation of China (No. 8166020210). 


\section{Authors' contributions:}

Hongfei Jiang: Writing, Conceptualization, Methodology, Software

Ren Gong.: Data curation, Original draft preparation.

Yaniqng Wu: Reviewing and Editing

Acknowledgements: No applicable

Declaration of Conflict of Interest: No applicable.

Availability of data and material: The data and material during the current study are available from the corresponding author on reasonable request.

\section{References}

1. Veerbeek JH, Brouwers L, Koster MP, Koenen SV, van Vliet EO, Nikkels PG, Franx A, van Rijn BB. Spiral artery remodeling and maternal cardiovascular risk: the spiral artery remodeling (SPAR) study[J]. J Hypertens. 2016;34(8):1570-7.

2. Zhou Z, Subramanian P, Sevilmis G, Globke B, Soehnlein O, Karshovska E, Megens R, Heyll K, Chun J, Saulnier-Blache JS, Reinholz M, van Zandvoort M, Weber C, Schober A. (2011) (2011) LipoproteinDerived Lysophosphatidic Acid Promotes Atherosclerosis by Releasing CXCL1?from the Endothelium. Cell Metab 13(5):592-600.

3. Pirillo A, Norata GD, Catapano AL. LOX-1, OxLDL, and atherosclerosis. Mediators Inflamm. 2013;24:152786.

4. Yang Y, Yang L, Liang X, Zhu Guofu. MicroRNA-155 Promotes Atherosclerosis Inflammation via Targeting SOCS1. Cell Physiol Biochem. 2015;36(4):1371-81.

5. Toba H, Cortez D, Lindsey ML, Chilton RJ. Applications of miRNA Technology for Atherosclerosis. Curr Atheroscler Rep. 2014;16(2):386.

6. Zhang Z, Pan X, Yang S, Ma A, Wang K, Wang Y, Li T, Liu S(2017) miR-155 Promotes ox-LDL-Induced Autophagy in Human Umbilical Vein Endothelial Cells. Mediators Inflamm 2017: 9174801.

7. Arai K, Jin G, Navaratna D, Lo EH. Brain angiogenesis in developmental and pathological processes: neurovascular injury and angiogenic recovery after stroke. Febs J. 2009;276(17):4644-52.

8. Geng Z, Wang J, Liu X, Cheng X, Zhang Y, Hu J, Li P, He Y, Song Y. MiR-129-5p-mediated Beclin-1 suppression inhibits endothelial cell autophagy in atherosclerosis. Am JTransl Res. 2016;8(4):188694.

9. Sun X, Icli B, Wara AK, Belkin N, He S, Kobzik L, Hunninghake GM, Vera MP, Blackwell TS, Baron RM, Feinberg MW. MicroRNA-181b regulates NF-??B-mediated vascular inflammation. J Clin Invest. 2012;122(6):1973-90. 
10. Liu X, Cheng Y, Yang J, Xu L, Zhang C. Cell-specific effects of miR-221/222 in vessels: Molecular mechanism and therapeutic application. J Mol Cell Cardiol. 2012;52(1):245-55.

11. Xin M, Small EM, Sutherland LB, Qi X, McAnally J, Plato CF, Richardson JA, Bassel-Duby R, Olson EN. MicroRNAs miR-143 and miR-145 modulate cytoskeletal dynamics and responsiveness of smooth muscle cells to injury. Genes Dev. 2009;23(18):2166-78.

12. Liu Y, Chen J, Dai M. Paeonol protects rat vascular endothelial cells from ox-LDL-induced injury in vitro via downregulating microRNA-21 expression and TNF-a release. Acta Pharmacol Sin. 2014;35(4):483-8.

13. Xu C, Shao Y, Xia T, Yang Y, Dai J, Luo L, Zhang X, Sun W, Song H, Xiao B, Guo J. IncRNA-AC130710 targeting by miR-129-5p is upregulated in gastric cancer and associates with poor prognosis. Tumor Biol. 2014;35(10):9701-6.

14. Long X, Zhou Y, Peng A, Zhang Z, Chen X, Chen W, Liu J, Huang S, Liu Z. Demethylation-mediated miR-129-5p up-regulation inhibits malignant phenotype of osteogenic osteosarcoma by targeting Homo sapiens valosin-containing protein (VCP). Tumor Biol. 2015;36(5):3799-806.

15. Feng J, Guo J, Wang J-P, et al. MiR-129-5p inhibits proliferation of gastric cancer cells through targeted inhibition on HMGB1 expression.[J]. Eur Rev Med Pharmacol Sci. 2020;24:3665-73.

16. Pang X, Zhang Y, Zhang S. High-mobility group box 1 is overexpressed in cervical carcinoma and promotes cell invasion and migration in vitro. Oncol Rep. 2017;37(2):831-40.

17. Chan $\mathrm{K}$, Ho HH, Lin M, Yen $\mathrm{CH}$, Huang C, Huang H, Wang CJ. Mulberry Water Extracts Inhibit Atherosclerosis through Suppression of the Integrin- $33 /$ Focal Adhesion Kinase Complex and Downregulation of Nuclear Factor KB Signaling in Vivo and in Vitro. J Agric Food Chem. 2014;62(39):9463.

18. Mehta D, Malik AB. Signaling mechanisms regulating endothelial permeability. Physiol Rev. 2006;86(1):279-367.

19. Koshman YE, Patel N, Chu M, lyengar R, Kim T, Ersahin C, Lewis W, Heroux A, Samarel AM. Regulation of Connective Tissue Growth Factor Gene Expression and Fibrosis in Human Heart Failure. J Card Fail. 2013;19(4):283-94.

20. Soltani A, Argani H, Rahimipour H, Soleimani F, Rahimi F, Kazerouni F. Oxidized LDL: As a risk factor for cardiovascular disease in renal transplantation. J Bras Nefrol. 2016;38(2):147-52.

21. Maiolino G, Rossitto G, Caielli P, Bisogni V, Rossi GP, Calo LA(2013) The Role of Oxidized Low-Density Lipoproteins in Atherosclerosis: The Myths and the Facts. Mediators Inflamm 2013:714653.

22. Li W, Zhi W, Liu F, He Z, Wang X, Niu X. Atractylenolide I restores HO-1 expression and inhibits OxLDL-induced VSMCs proliferation, migration and inflammatory responses in vitro. Exp Cell Res. 2017;353(1):26-34.

23. Zhang L, Cheng H, Yue Y, Li S, Zhang D, He R. H19 knockdown suppresses proliferation and induces apoptosis by regulating miR-148b/WNT/ $\beta$-catenin in ox-LDL-stimulated vascular smooth muscle cells. J Biomed Sci. 2018;25(1):11. 
24. Madrigal-Matute J, Rotllan N, Aranda JF, Fernandez-Hernando C. MicroRNAs and Atherosclerosis. Curr Atheroscler Rep. 2013;15(5):322.

25. Florijn BW, Bijkerk R, van der Veer EP, van Zonneveld AJ. Gender and cardiovascular disease: Are sexbiased miRNA networks a driving force behind heart failure with preserved ejection fraction in women? Cardiovasc Res. 2018;114(2):210-25.

26. Luo J, Chen J, He L. mir-129-5p Attenuates Irradiation-Induced Autophagy and Decreases Radioresistance of Breast Cancer Cells by Targeting HMGB1. Med Sci Monit. 2015;21:4122-9.

27. Han JS, Kim K, Lee M. A high mobility group B-1 box A peptide combined with an artery wall binding peptide targets delivery of nucleic acids to smooth muscle cells. J Cell Biochem. 2009;107(1):16370 .

\section{Figures}

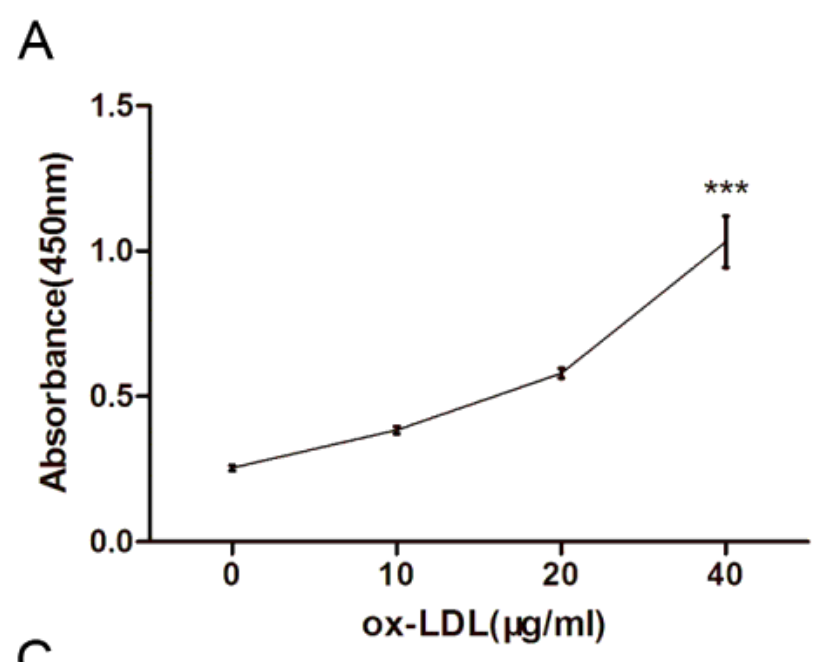

B
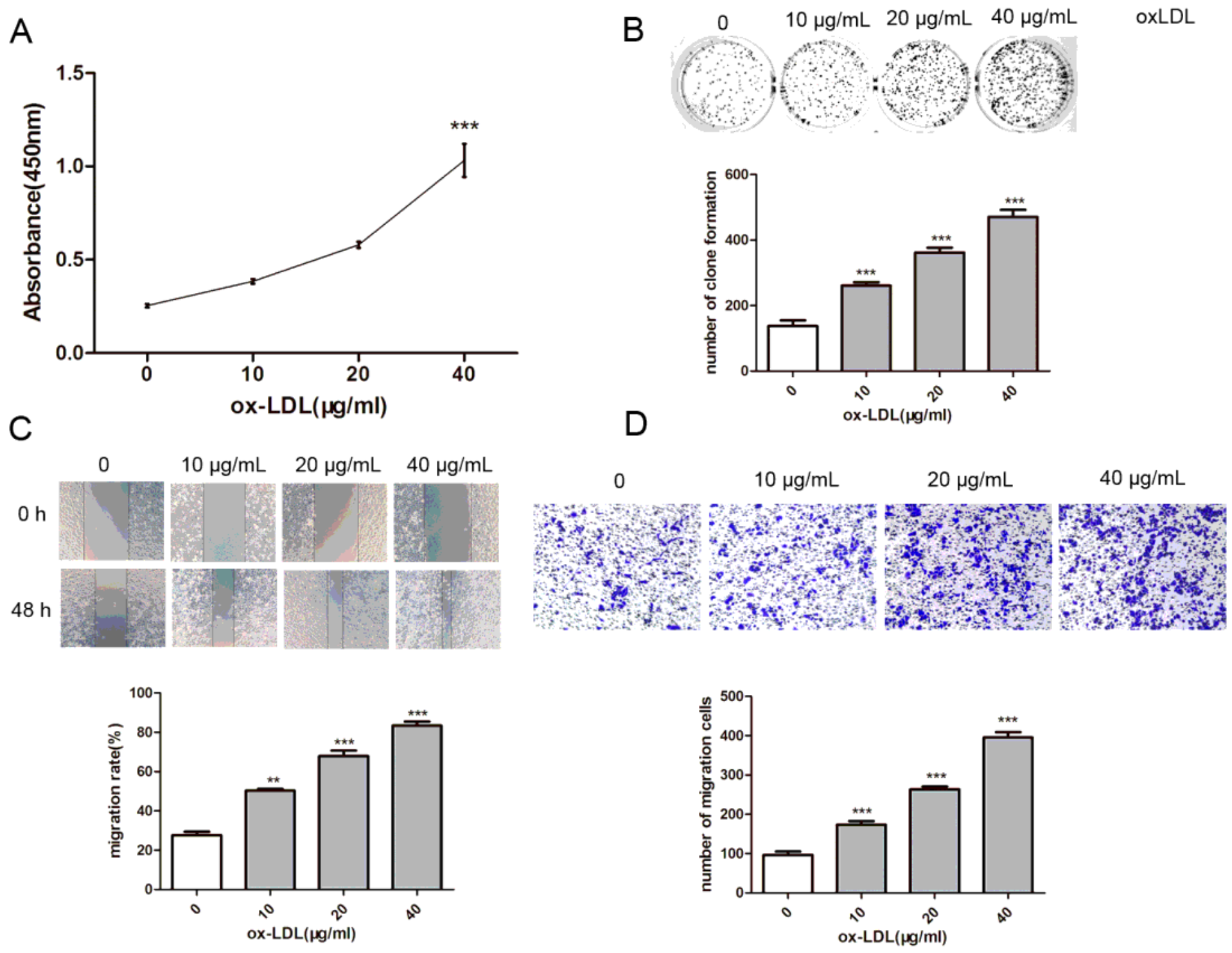

Figure 1 
A7r5 cells induced by ox-LDL showed increased proliferation and migration. (A) Effects of different doses of ox-LDL on A7r5 cells viability were measured using CCK-8 assay. (B) Clonogenicity was detected by colony formation assay. (C) Wound closure of cells was assessed using wound healing assay. (D) Migration of cells was performed through transwell assay. ${ }^{* *} \mathrm{P}<0.01,{ }^{*} * \mathrm{P}<0.001, \mathrm{n}=3$.

A

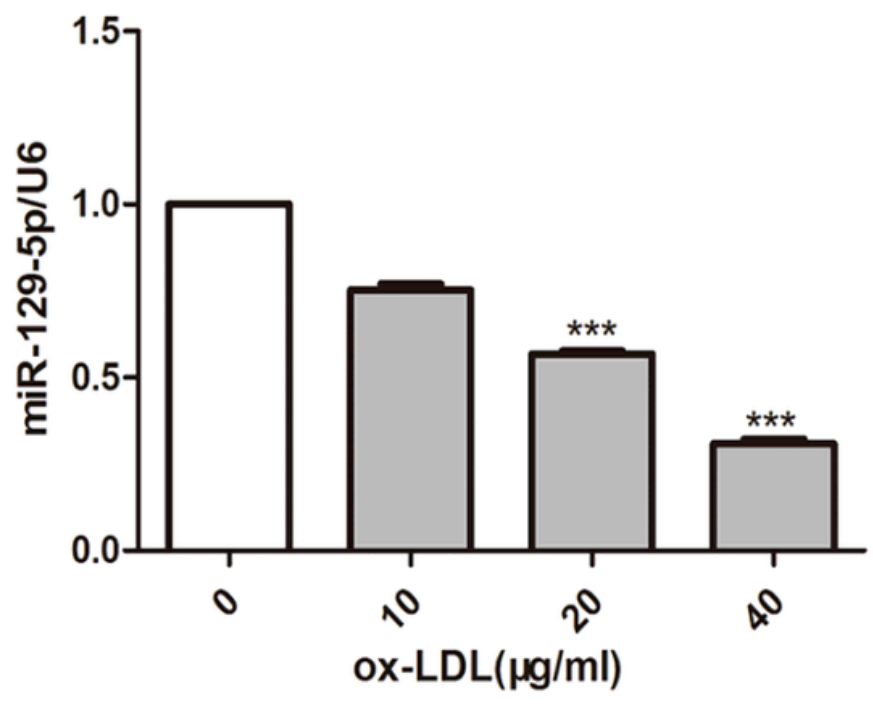

B

C
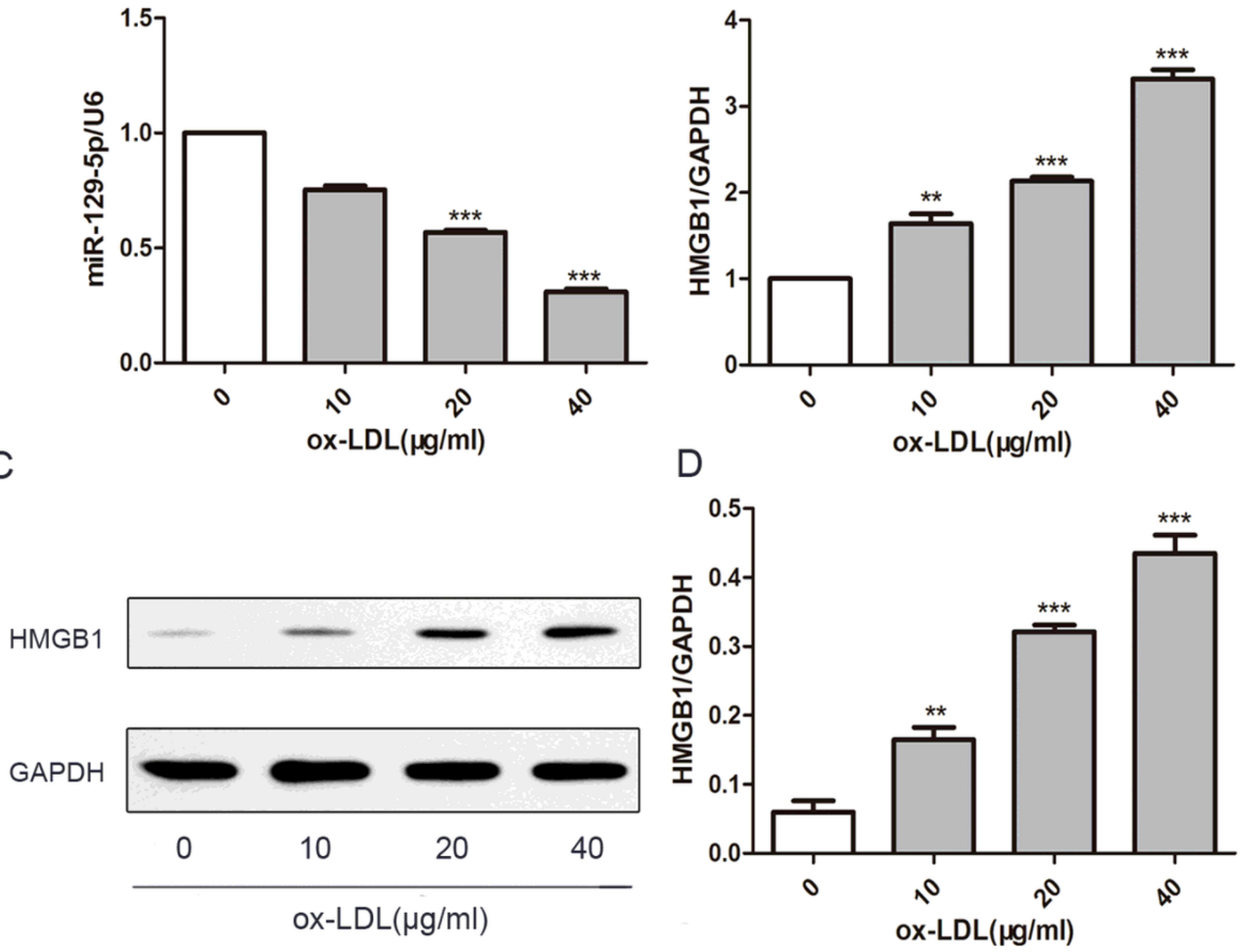

Figure 2

miR-129-5p was downregulated in ox-LDL induced A7r5 cells and HMGB1 was up-regulated in ox-LDL induced A7r5 cells. (A) QRT-PCR assays determine the expression of miR-129-5p in A7r5 cells induced by $0,10,20,40 \mu \mathrm{g} / \mathrm{mL}$ ox-LDL. (B) QRT-PCR assays determine the expression of HMGB1 in A7r5 cells induced by $0,10,20,40 \mu \mathrm{g} / \mathrm{mL}$ ox-LDL. (C) Western blot assays determine the expression of HMGB1. Protein levels in A7r5 cells infused with $0,10,20,40 \mu \mathrm{g} / \mathrm{mL}$ ox-LDL. (D) The quantization diagrams of the protein expression levels of HMGB1 in A7r5 cells. ${ }^{*} P<0.01,{ }^{* \star *} P<0.001, n=3$. 

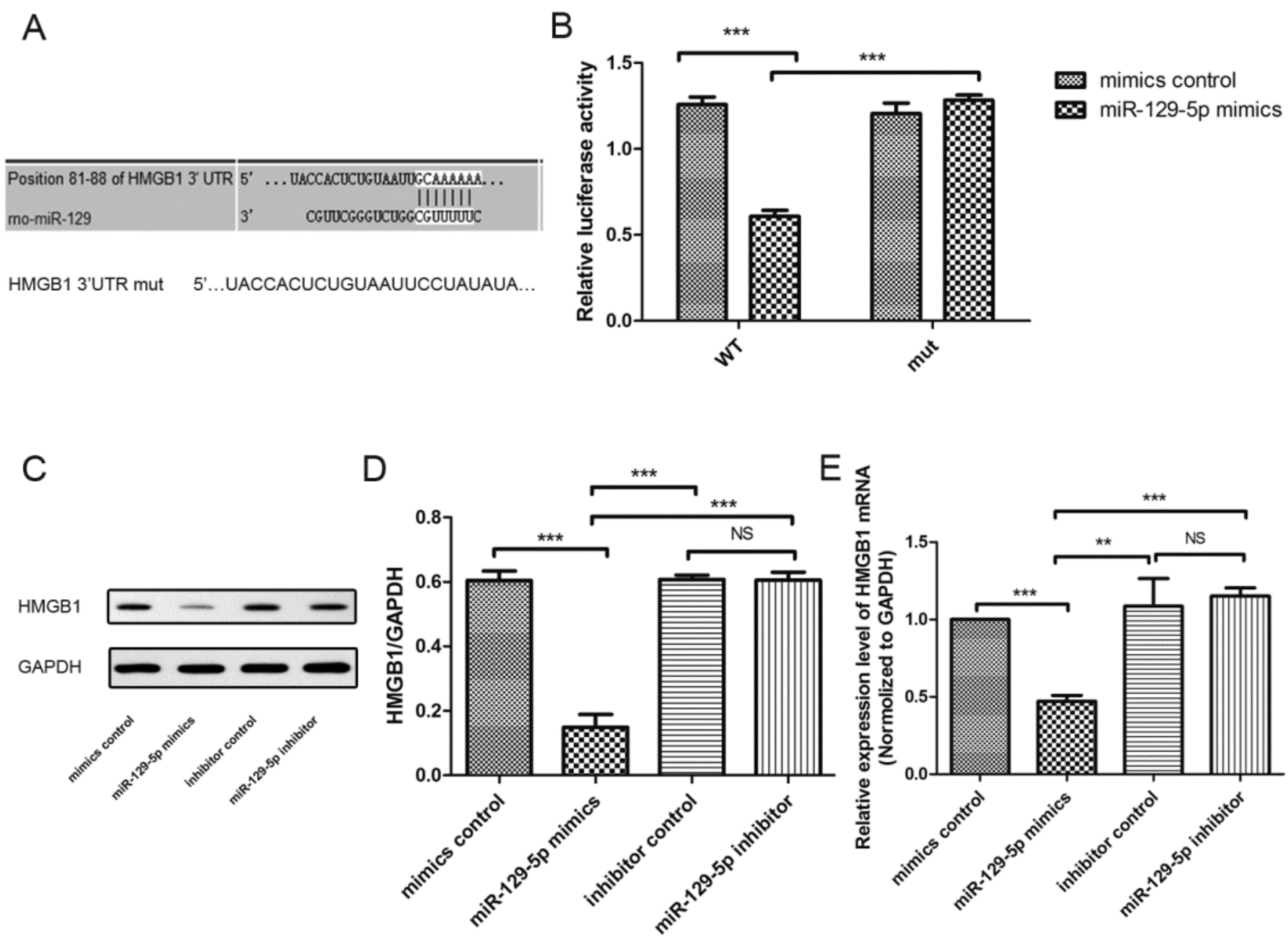

Figure 3

miR-129-5p directly targets HMGB1 in A7r5 cells. (A) Bioinformatics analyses showing that miR-129-5p targets 3'UTR of HMGB1 mRNA at one binding site of base pair 81st to 88th. (B) The mutant reporter of HMGB1 was constructed. Then A7r5 cells were co-transfected with either miR-129-5p mimics or mimics control. The relative luciferase activity was detected after $48 \mathrm{~h}$. (C) Western blotting assay was used to detect HMGB1 protein levels with miR-129-5p over-expressed in A7r5 cells. ${ }^{* *} \mathrm{P}<0.001, n=3$. 


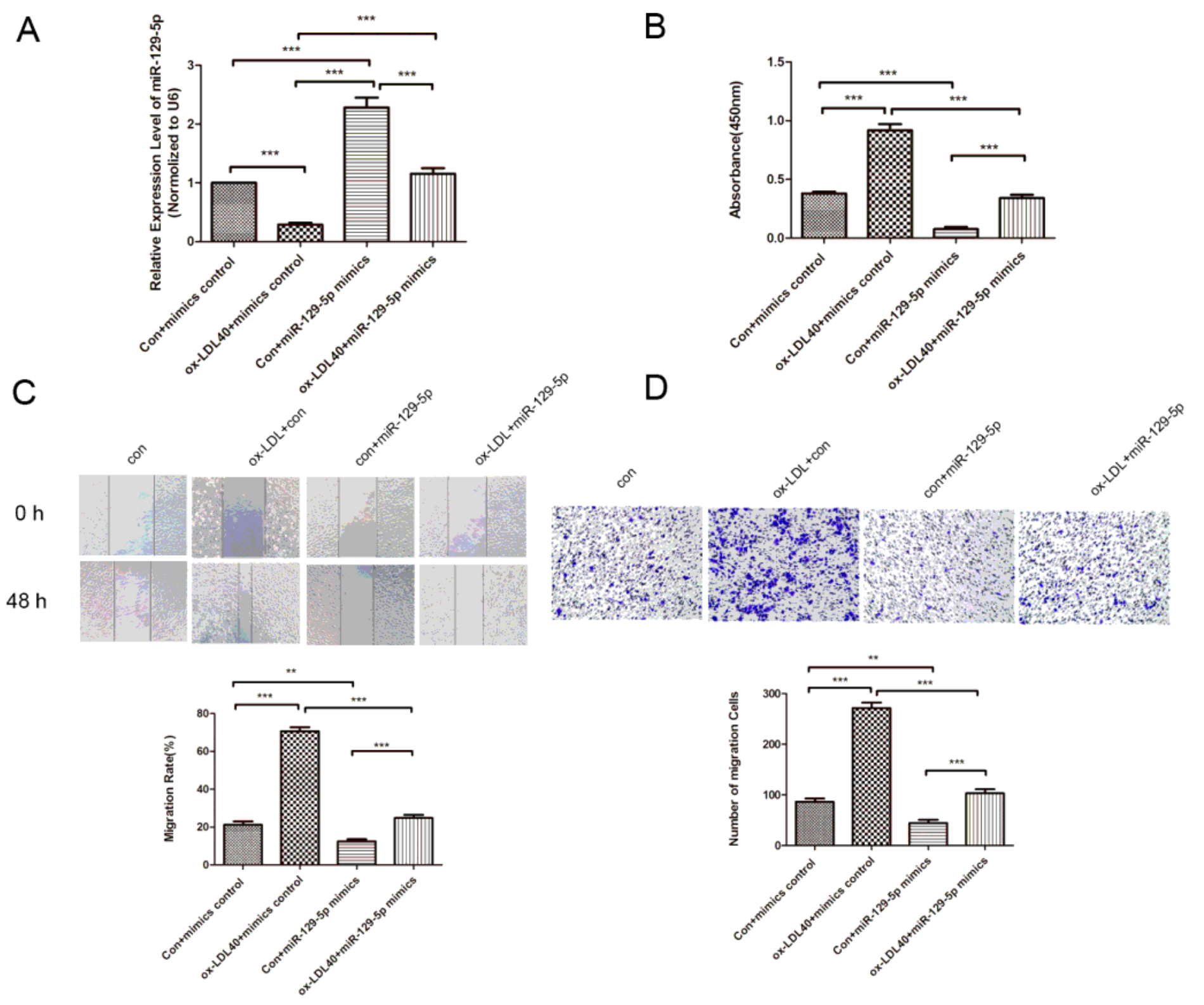

Figure 4

miR-129-5p inhibits ox-LDL-induced A7r5 cells proliferation and Migration. (A) QRT-PCR assays determine the expression of miR-129-5p mRNA in A7r5 cells. (B) $48 \mathrm{~h}$ later, effects of different treatment on A7r5 cells viability were measured using CCK-8 assay. (B) $48 \mathrm{~h}$ later, effects of different treatment on A7r5 cells viability were detected using CCK-8 assay. (C) Wound closure of cells was detected using wound healing assay after 48 h. (D) Migration of cells was performed through transwell assay. ${ }^{\star *} P<0.01,{ }^{* \star \star} P<0.001$, $n=3$. 


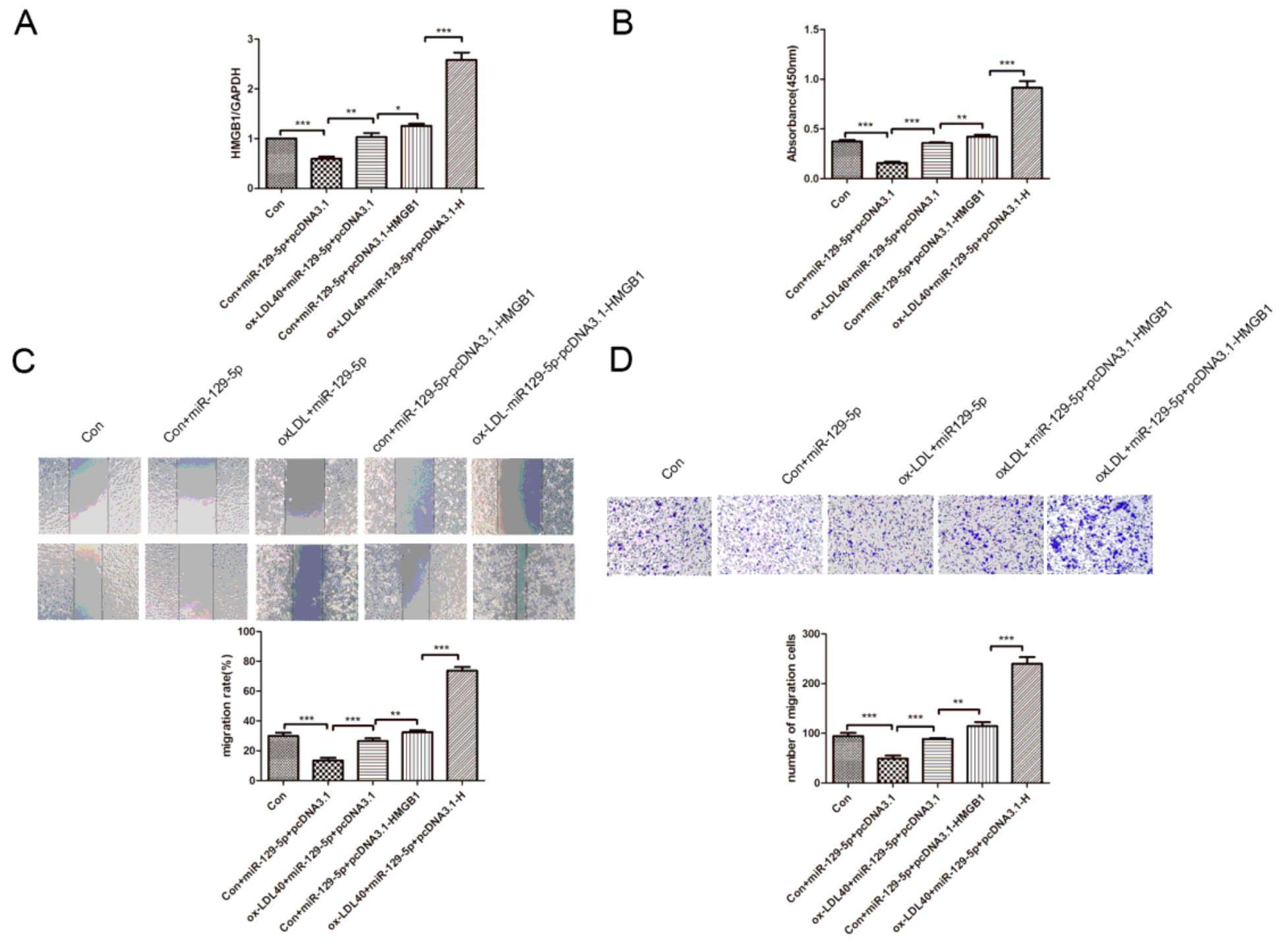

Figure 5

Overexpression of HMGB1 rescued the result of miR-129-5p inhibit ox-LDL-induced A7r5 cells proliferation and migration. (A) QRT-PCR assays determine the expression of HMGB1 mRNA in A7r5 cells. And the groups are as follows: Control group, con+miR129-5p+pcDNA3.1, 40 $\mathrm{g} / \mathrm{mL}$ ox-LDL+miR1295p+pcDNA3.1, con+miR-129-5p+pcDNA3.1-HMGB1, 40 $\mathrm{g} / \mathrm{mL}$ ox-LDL+miR129-5p+pcDNA3.1-HMGB1. (B) $48 \mathrm{~h}$ later, effects of different treatment on A7r5 cells viability were measured using CCK-8 assay. (C) Wound closure of cells was detected using wound healing assay after $48 \mathrm{~h}$. (D) Migration of cells was performed through Transwell assay. ${ }^{* \star} \mathrm{P}<0.01,{ }^{* \star *} \mathrm{P}<0.001, \mathrm{n}=3$. 
$A$
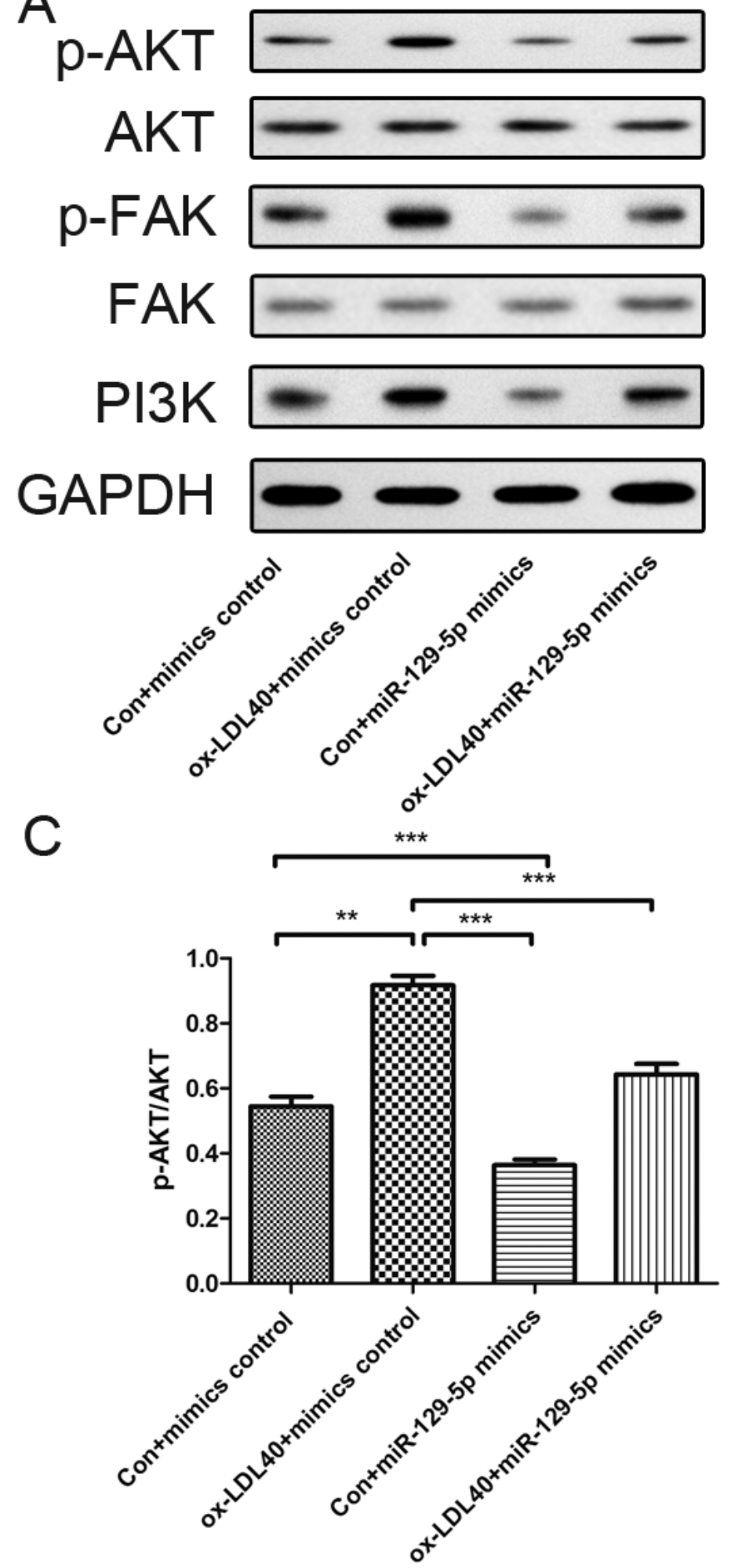

$B$

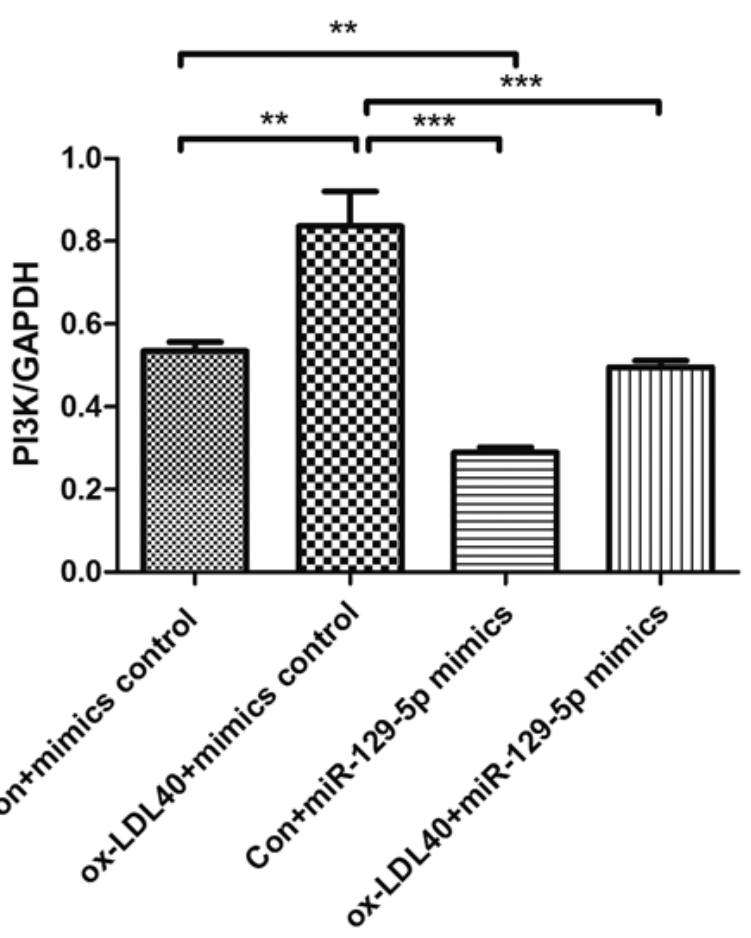

D

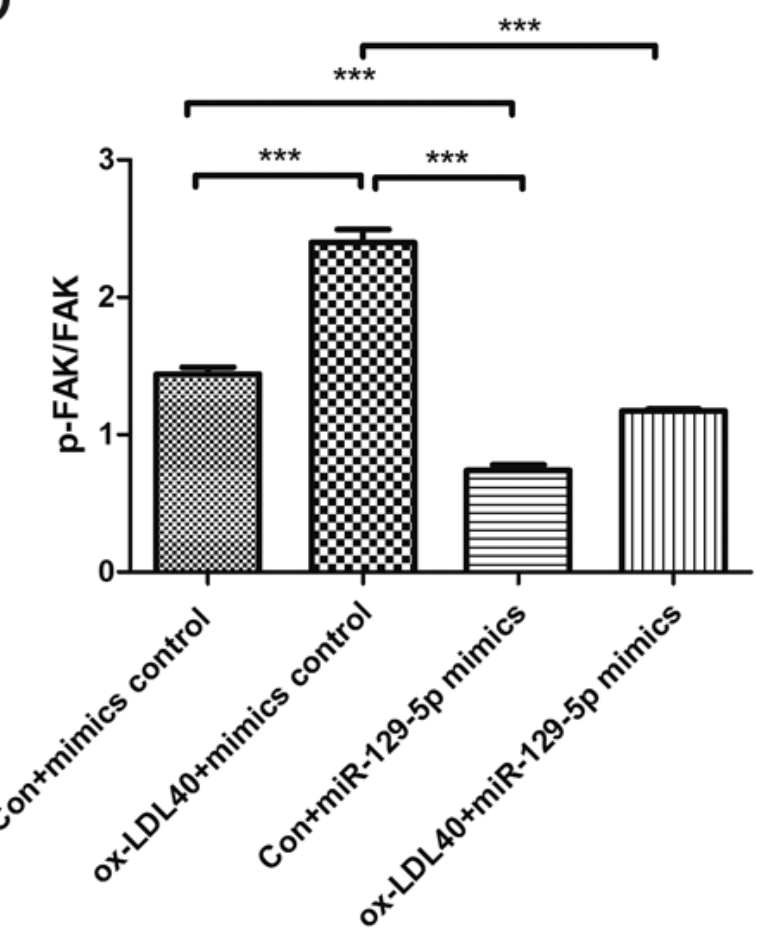

Figure 6

The effect of miR-129-5p on protein expression PI3K and phosphorylation of FAK and Akt. Western blot assays determine the phosphorylation of FAK, Akt, and PI3K. (A). Protein levels in A7r5 cells infused with mimics, $40 \mu \mathrm{g} / \mathrm{mL}$ ox-LDL + mimics control cells, miR-129-5p+mimics control and $40 \mu \mathrm{g} / \mathrm{mL}$ oxLDL+miR-129-5p mimics. (B, C, D) The quantization diagrams of the protein expression levels of PI3K, $p$ Akt and $p$-FAK respectively. ${ }^{* * P}<0.01,{ }^{*} * * P<0.001, n=3$. 